\title{
NRQCD analysis of charmonium production with pion and proton beams at fixed-target energies
}

\author{
Chia-Yu Hsieh ${ }^{\mathrm{a}, \mathrm{b}, 1}$, Yu-Shiang Lian ${ }^{\mathrm{a}, \mathrm{c}, 1}$, Wen-Chen Chang ${ }^{\mathrm{a}, *}$, Jen-Chieh Peng ${ }^{\mathrm{d}}$, Stephane Platchkov ${ }^{\mathrm{e}}$, and \\ Takahiro Sawada ${ }^{\mathrm{f}}$ \\ ${ }^{a}$ Institute of Physics, Academia Sinica, Taipei 11529, Taiwan \\ ${ }^{b}$ Department of Physics, National Central University, 300 Jhongda Road, Jhongli 32001, Taiwan. \\ ${ }^{c}$ Department of Physics, National Kaohsiung Normal University, Kaohsiung County 824, Taiwan \\ ${ }^{d}$ Department of Physics, University of Illinois at Urbana-Champaign, Urbana, Illinois 61801, USA \\ ${ }^{e}$ IRFU, CEA, Université Paris-Saclay, 91191 Gif-sur-Yvette, France \\ ${ }^{f}$ Department of Physics, Osaka City University, Osaka 558-8585, Japan
}

\begin{abstract}
We present an analysis of hadroproduction of $J / \psi$ and $\psi(2 S)$ at fixed-target energies in the framework of non-relativistic QCD (NRQCD). Using both pion- and proton-induced data, a new determination of the color-octet long-distance matrix elements (LDMEs) is obtained. Compared with previous results, the contributions from the $q \bar{q}$ and color-octet processes are significantly enhanced, especially at lower energies. A good agreement between the pion-induced $J / \psi$ production data and NRQCD calculations using the newly obtained LDMEs is achieved. We find that the pion-induced charmonium production data are sensitive to the gluon density of pions, and favor pion PDFs with relatively large gluon contents at large $x$.

Keywords: charmonium production, pion PDFs, NRQCD, color-octet matrix elements, gluon
\end{abstract}

\section{Introduction}

Since the discovery of the $J / \psi$ resonance in 1974 , the study of $c \bar{c}$ and $b \bar{b}$ quarkonium states has significantly improved our understanding of the strong interaction [1, 2, 3, 4, 5]. Production of heavy-quark bound states offers important testing grounds for both perturbative and nonperturbative aspects of QCD. From the experimental perspective, quarkonia are readily detected through their dilepton decay, and the associated production cross sections are large compared to other hard processes. Since hadroproduction of quarkonium receives important contribution form gluon-gluon fusion it could serve as a tool for accessing the gluon distributions of interacting hadrons, and particularly for the unstable pion. Our current knowledge on the pion parton distribution functions ( $\mathrm{PDFs}$ ) mainly comes from pion-induced Drell-Yan data. As the Drell-Yan cross sections are dominated by $\bar{q}-q$ annihilation, they essentially probe the valence quark

\footnotetext{
* Corresponding author

Email address: changwc@phys.sinica.edu.tw (Wen-Chen Chang)

${ }^{1}$ These authors contributed equally to this work.
} 
distribution in the pion, but leave the sea and the gluon distributions unconstrained. The mounting interest in the pion PDFs is reflected in many publications in the past few years. The pion PDFs were extracted using new and refined global analyses [6, 7, 8], or calculated from new theoretical developments, such as the continuum Dyson-Schwinger Equations [9, 10], light-front holographic QCD [11], and light-front Hamiltonian [12]. First lattice QCD results on the $x$-dependence of pion valence structure are also becoming available [13, 14, 15, 16, 17, 18.

A significant amount of pion-induced charmonium data has been collected in the past. Recently, we investigated the sensitivity of the fixed-target $J / \psi$ production data to the pion PDFs [19]. The pion-induced $J / \psi$ data on hydrogen and several light-mass nuclear targets were compared to next-to-leading-order (NLO) Color Evaporation Model (CEM) [20] calculations using the available global-fit parametrizations of the pion PDFs. Over the energy range considered, the calculations with pion PDFs determined by SMRS [21], GRV [22, JAM [6] and xFitter [7, are all in reasonable agreement with the total cross sections data. In contrast, for the longitudinal momentum $x_{F}$ distributions, we found that the agreement between data and calculations strongly depends on the magnitude and shape of the gluon distribution in the pion. The data favor pion PDFs with larger gluon strength at large $x$.

To examine a possible model dependence of our previous observations [19], we extend our work to a more elaborate formalism for quarkonia production, namely, the non-relativistic QCD (NRQCD) [23] approach. This approach is based on a factorization of the heavy-quark pair $Q \bar{Q}$ production and its subsequent hadronization. The production of the $Q \bar{Q}$ pair proceeds through a short-distance partonic interaction, calculated using perturbative QCD. The probability of hadronization of a $Q \bar{Q}$ pair into some quarkonium bound state depends on its spin, color and angular momentum. Being of a non-perturbative nature, the hadronization is described by the associated long-distance matrix elements (LDMEs). The LDMEs, assumed to be universal, are extracted from the experimental data. The values for the color-singlet (CS) LDMEs can be obtained from the decay widths of charmonia or model calculations, while the color-octet (CO) ones are determined from fits to data of transverse-momentum $\left(p_{T}\right)$ differential cross sections, polarization, and $p_{T}$-integrated cross sections of charmonium production.

The NRQCD approach is based on the assumption that the factorization holds [23]. However, for low $p_{T}$ additional effects might have large contributions. Analysis of fixed-target data could provide hints for such effects by assessing the universality of the LDMEs. Such study, focusing exclusively on charmonium hadroproduction from fixed-target experiments, was reported by Beneke and Rothstein [24]. The calculation was performed at partial next-to-leading order (up to $\mathcal{O}\left(\alpha_{s}^{3}\right)$ ). Some LDMEs were taken from fits to collider data of $p_{T}$-differential cross sections [25, 26] while others were fitted to the proton-induced cross section. The resulting LDMEs were used to calculate the pion-induced total cross sections. The calculation was found to lie systematically below the data, for both $J / \psi$ and $\psi(2 S)$ production. The failure in describing the pion-induced data was speculated to be due to either inaccurate gluon distributions of pion PDFs or a 
difference in the higher twist effects between the proton and the pion data [24].

More recently, Maltoni et al. 27] re-analyzed the data of $J / \psi$ and $\psi(2 S)$ fixed-target proton-induced cross sections. The pion-induced data were not included in the analysis. Their NRQCD calculation was performed at NLO using the formalism described in Ref. [28]. The initial CO parameters were taken from the Tevatron data [29, 30, but the S-wave CO LDMEs for $J / \psi$ and $\psi(2 S)$ were allowed to vary. A good description of the data was achieved, at the expense of a strong reduction of the color-octet LDMEs by a factor of 5 to 10, depending on the proton PDFs used.

By using the NRQCD formalism in the fixed-target domain, the primary motivation of our present work is twofold. First, we aim at the determination of a new set of LDMEs that can reproduce both pion and proton-induced $\mathrm{J} /$ psi production cross sections. As the pion contains a valence antiquark, the $J / \psi$ cross section for a pion beam has a larger $q \bar{q}$ contribution than that for a proton beam. Thereby, the pion-induced data provide a stronger constraint on the LDMEs that are responsible for the $q \bar{q}$ part of the cross section. Second, we use this new set of LDMEs to explore the sensitivity of the pion-induced charmonium data to the parametrizations of the pion PDFs. The most recent global fits [6, 7] lead to pion gluon distributions which significantly differ from the older pion PDF determinations [21, 22, at large and medium parton momenta [19]. According to our previous results [19], these new sets of pion PDFs bring worse agreement between the CEM calculations and the data. It is important to validate these observations with a NRQCD calculation.

To perform this study, we adopt the NRQCD framework explicitly formulated in Ref. [24]. We start with a comparison of NRQCD calculations with the total cross section data for $J / \psi$ and $\psi(2 S)$ productions with proton and pion beams. We then vary the CO LDMEs for $J / \psi$ and $\psi(2 S)$ production to achieve a consistent description of both proton and pion data. In addition, we use the new LDMEs to explore the sensitivity of the pion-induced $J / \psi$ data to the gluon distribution in the pion. This paper is organized as follows. In Sec. 2, we introduce the NRQCD framework for the calculations of charmonia cross sections in hadroproduction. A new fit to the fixed-target $J / \psi$ and $\psi(2 S)$ production data to determine a new set of LDMEs is presented in Sec. 3. We further discuss several findings from the present study in Sec. 4, followed by a summary in Sec. 5

\section{Charmonium Production and NRQCD}

In NRQCD, the differential cross section as a function of Feynman $x\left(x_{F}\right), d \sigma / d x_{F}$ for a charmonium state $H\left(H=J / \psi, \psi(2 S)\right.$, or $\left.\chi_{c J}\right)$ from the $h N,(h=p, \bar{p}$, or $\pi)$ collisions, where $h$ is the beam hadron 
and $N$ the target nucleon, is 31 ]

$$
\begin{gathered}
\frac{d \sigma^{H}}{d x_{F}}=\sum_{i, j=q, \bar{q}, G} \int_{0}^{1} d x_{1} d x_{2} \delta\left(x_{F}-x_{1}+x_{2}\right) \\
\times f_{i}^{h}\left(x_{1}, \mu_{F}\right) f_{j}^{N}\left(x_{2}, \mu_{F}\right) \hat{\sigma}[i j \rightarrow H]\left(x_{1} P_{h}, x_{2} P_{N}, \mu_{F}, \mu_{R}, m_{c}\right), \\
\hat{\sigma}[i j \rightarrow H]=\sum_{n} C_{c \bar{c}[n]}^{i j}\left(x_{1} P_{h}, x_{2} P_{N}, \mu_{F}, \mu_{R}, m_{c}\right) \times\left\langle\mathcal{O}_{n}^{H}\left[{ }^{2 S+1} L_{J}\right]\right\rangle \\
x_{F}=2 p_{L} / \sqrt{s}, x_{1,2}=\frac{\sqrt{x_{F}^{2}+4 M_{c \bar{c}}{ }^{2} / s} \pm x_{F}}{2}
\end{gathered}
$$

where $i$ and $j$ label the type of interacting partons (gluons, quarks and antiquarks), and the $c \bar{c}$ pair is denoted by its color $(n)$, spin $(S)$, orbital angular momentum $(L)$ and total angular momentum $(J)$. Here $m_{c}$ and $M_{c \bar{c}}$ are the charm quark and $c \bar{c}$ pair masses, $f^{h}$ and $f^{N}$ are the incoming hadron and the target nucleon parton distribution functions, evaluated at their respective Bjorken- $x$ values, $x_{1}$ and $x_{2}$. The $\mu_{F}$ and $\mu_{R}$ are the factorization and renormalization scales. The total cross sections are obtained by integrating over $x_{F}$.

The production cross section $\hat{\sigma}[i j \rightarrow H]$ is the sum of the products of the short-distance coefficients $C_{c \bar{c}[n]}^{i j}$, calculated perturbatively in powers of $\alpha_{s}\left(\mu_{R}\right)$, and the nonperturbative parameters, LDMEs $\left\langle\mathcal{O}_{n}^{H}\left[{ }^{2 S+1} L_{J}\right]\right\rangle$. The coefficients $C_{c \bar{c}[n]}^{i j}$ describe the production of a $c \bar{c}$ pair in a specific spin-color state $[n]$, while the parameters $\left\langle\mathcal{O}_{n}^{H}\left[{ }^{2 S+1} L_{J}\right]\right\rangle$ account for the hadronization of the $c \bar{c}[n]$ pair into the charmonium state $H$. The LDMEs are mainly determined by a fit to data and assumed to be universal, independent of beam or target hadrons and the energy scale.

In this work, we use the expressions given in Ref. [24] for computation of $J / \psi, \psi(2 S)$, and $\chi_{c J}$ production via $G G, q \bar{q}$ and $q G$ processes. The scattering processes $q \bar{q} \rightarrow Q \bar{Q}$ and $G G \rightarrow Q \bar{Q}$ at $\mathcal{O}\left(\alpha_{s}^{2}\right)$ produce $Q \bar{Q}$ pairs in an S-wave CO state or P-wave CS state. Starting from $\mathcal{O}\left(\alpha_{s}^{3}\right)$, the $\mathrm{S}$-wave or P-wave CS $Q \bar{Q}$ pairs can be produced through the scattering processes $G G \rightarrow Q \bar{Q} G$ and $q G \rightarrow Q \bar{Q} q$ [26]. We use this NRQCD framework for calculating the total cross sections of $J / \psi$ and $\psi(2 S)$ production in hadronic collision.

The relationship of LDMEs to the scattering processes for each charmonium state is summarized in Table 1. For the $q \bar{q}$ process, the $c \bar{c}$ pairs are produced at $\mathcal{O}\left(\alpha_{s}^{2}\right)$ in color octet states, which then hadronize into various charmonium states with the LDMEs $\left\langle\mathcal{O}_{8}^{H}\left[{ }^{3} S_{1}\right]\right\rangle$. For the $G G$ process, both $J / \psi$, and $\psi(2 S)$ can be produced from either the $\operatorname{CO} c \bar{c}$ at $\mathcal{O}\left(\alpha_{s}^{2}\right)$ or the $\operatorname{CS} c \bar{c}$ at $\mathcal{O}\left(\alpha_{s}^{3}\right)$. The corresponding hadronization LDMEs for the CO and CS states are $\Delta_{8}^{H}=\left\langle\mathcal{O}_{8}^{H}\left[{ }^{1} S_{0}\right]\right\rangle+3 / m_{c}^{2}\left\langle\mathcal{O}_{8}^{H}\left[{ }^{3} P_{0}\right]\right\rangle+4 /\left(5 m_{c}^{2}\right)\left\langle\mathcal{O}_{8}^{H}\left[{ }^{3} P_{2}\right]\right\rangle$ and $\left\langle\mathcal{O}_{1}^{H}\left[{ }^{3} S_{1}\right]\right\rangle$, respectively. The $\chi_{c 0}, \chi_{c 1}$ and $\chi_{c 2}$ can be produced from CS $c \bar{c}$ states in the $G G$ process at either $\mathcal{O}\left(\alpha_{s}^{2}\right)$ $\left(\chi_{c 0}, \chi_{c 2}\right)$ or $\mathcal{O}\left(\alpha_{s}^{3}\right)\left(\chi_{c 1}\right)$ with the corresponding hadronization LDMEs $\left\langle\mathcal{O}_{1}^{H}\left[{ }^{3} P_{0}\right]\right\rangle / m_{c}^{2},\left\langle\mathcal{O}_{1}^{H}\left[{ }^{3} P_{1}\right]\right\rangle / m_{c}^{2}$ and $\left\langle\mathcal{O}_{1}^{H}\left[{ }^{3} P_{2}\right]\right\rangle / m_{c}^{2}$. At $\mathcal{O}\left(\alpha_{s}^{3}\right)$, the $q G$ process can contribute to the production of $\chi_{c 1}$ via the CS $c \bar{c}$ state which hadronizes with the LDME $\left\langle\mathcal{O}_{1}^{H}\left[{ }^{3} P_{1}\right]\right\rangle / m_{c}^{2}$. 


\begin{tabular}{|c|c|c|c|}
\hline$H$ & $q \bar{q}$ & $G G$ & $q G$ \\
\hline$J / \psi, \psi(2 S)$ & $\left\langle\mathcal{O}_{8}^{H}\left[{ }^{3} S_{1}\right]\right\rangle\left(\mathcal{O}\left(\alpha_{s}^{2}\right)\right)$ & $\begin{array}{c}\Delta_{8}^{H *}\left(\mathcal{O}\left(\alpha_{s}^{2}\right)\right) \\
\end{array}$ & \\
\hline$\chi_{c 0}$ & $\left.\left\langle\mathcal{O}_{8}^{H}\left[{ }^{3} S_{1}\right]\right\rangle\left(\mathcal{O} S_{1}\right]\right\rangle\left(\mathcal{O}\left(\alpha_{s}^{3}\right)\right)$ & $\left\langle\mathcal{O}_{1}^{H}\left[{ }^{3} P_{0}\right]\right\rangle\left(\mathcal{O}\left(\alpha_{s}^{2}\right)\right)$ & \\
\hline$\chi_{c 1}$ & $\left\langle\mathcal{O}_{8}^{H}\left[{ }^{3} S_{1}\right]\right\rangle\left(\mathcal{O}\left(\alpha_{s}^{2}\right)\right)$ & $\left\langle\mathcal{O}_{1}^{H}\left[{ }^{3} P_{1}\right]\right\rangle\left(\mathcal{O}\left(\alpha_{s}^{3}\right)\right)$ & $\left\langle\mathcal{O}_{1}^{H}\left[{ }^{3} P_{1}\right]\right\rangle\left(\mathcal{O}\left(\alpha_{s}^{3}\right)\right)$ \\
\hline$\chi_{c 2}$ & $\left\langle\mathcal{O}_{8}^{H}\left[{ }^{3} S_{1}\right]\right\rangle\left(\mathcal{O}\left(\alpha_{s}^{2}\right)\right)$ & $\left\langle\mathcal{O}_{1}^{H}\left[{ }^{3} P_{2}\right]\right\rangle\left(\mathcal{O}\left(\alpha_{s}^{2}\right)\right)$ & \\
\hline
\end{tabular}

Table 1: Relationship of LDMEs and the associated orders of $\alpha_{s}$ to the scattering processes for various charmonium states in the NRQCD framework of Ref. [24]. ${ }^{*}: \Delta_{8}^{H}=\left\langle\mathcal{O}_{8}^{H}\left[{ }^{1} S_{0}\right]\right\rangle+\frac{3}{m_{c}^{2}}\left\langle\mathcal{O}_{8}^{H}\left[{ }^{3} P_{0}\right]\right\rangle+\frac{4}{5 m_{c}^{2}}\left\langle\mathcal{O}_{8}^{H}\left[{ }^{3} P_{2}\right]\right\rangle$

The number of independent LDMEs is further reduced by applying the spin symmetry relations [24, 27]:

$$
\begin{aligned}
\left\langle\mathcal{O}_{8}^{J / \psi, \psi(2 S)}\left[{ }^{3} P_{J}\right]\right\rangle & =(2 J+1)\left\langle\mathcal{O}_{8}^{J / \psi, \psi(2 S)}\left[{ }^{3} P_{0}\right]\right\rangle \text { for } J=2 \\
\left\langle\mathcal{O}_{8}^{\chi_{c J} J}\left[{ }^{3} S_{1}\right]\right\rangle & =(2 J+1)\left\langle\mathcal{O}_{8}^{\chi_{c 0}}\left[{ }^{3} S_{1}\right]\right\rangle \text { for } J=1,2 \\
\left\langle\mathcal{O}_{1}^{\chi_{c J} J}\left[{ }^{3} P_{J}\right]\right\rangle & =(2 J+1)\left\langle\mathcal{O}_{1}^{\chi_{c 0}}\left[{ }^{3} P_{0}\right]\right\rangle \text { for } J=1,2 .
\end{aligned}
$$

The best-fit LDMEs for the description of proton-induced $J / \psi$ and $\psi(2 S)$ production from Ref. [24] are summarized in Table 2. In Ref. [24], the CS LDMEs $\left\langle\mathcal{O}_{1}^{H}\left[{ }^{3} S_{1}\right]\right\rangle$ for $J / \psi$ and $\psi(2 S)$, and $\left\langle\mathcal{O}_{1}^{H}\left[{ }^{3} P_{0}\right]\right\rangle$ for $\chi_{c 0}$, are given by the potential models [25], and the CO LDMEs $\left\langle\mathcal{O}_{8}^{H}\left[{ }^{3} S_{1}\right]\right\rangle$ for $J / \psi, \psi(2 S)$ and $\chi_{c}$, are taken from the fits to Tevatron collider data of $p_{T}$ spectra [26]. The $\Delta_{8}^{H}$ parameters for the individual $J / \psi$ and $\psi(2 S)$ production were determined by a fit of NRQCD calculation to the proton-induced data.

A later study by Maltoni et al. [27] was performed with a full NLO calculation [28]. Starting with the LDMEs determined from the collider data of $p_{T}$ differential cross sections [29, 30, an additional reduction factor of about 0.1 for $\left\langle\mathcal{O}_{8}^{H}\left[{ }^{3} S_{1}\right]\right\rangle$ and $\left\langle\mathcal{O}_{8}^{H}\left[{ }^{1} S_{0}\right]\right\rangle$ of $J / \psi$ and $\psi(2 S)$, was required in the calculations to achieve a good description of fixed-target proton-induced data.

The CS LDMEs used in these two studies are either identical or very similar, as they were obtained from a potential model $[25]$. The $\mathrm{CO}\left\langle\mathcal{O}_{8}^{H}\left[{ }^{3} S_{1}\right]\right\rangle$ for $\chi_{c 0}$ are nearly identical. In contrast, the remaining CO LDMEs obtained in Ref. [24] are about a factor of 1.5-5 larger than those determined in Ref. 27].

\begin{tabular}{|c|c|c|c|c|}
\hline$H$ & $\left\langle\mathcal{O}_{1}^{H}\left[{ }^{3} S_{1}\right]\right\rangle$ & $\left\langle\mathcal{O}_{1}^{H}\left[{ }^{3} P_{0}\right]\right\rangle / m_{c}{ }^{2}$ & $\left\langle\mathcal{O}_{8}^{H}\left[{ }^{3} S_{1}\right]\right\rangle$ & $\Delta_{8}^{H}$ \\
\hline$J / \psi$ & 1.16 & & $6.6 \times 10^{-3}$ & $3 \times 10^{-2}$ \\
$\psi(2 S)$ & 0.76 & & $4.6 \times 10^{-3}$ & $5.2 \times 10^{-3}$ \\
$\chi_{c 0}$ & & 0.044 & $3.2 \times 10^{-3}$ & \\
\hline
\end{tabular}

Table 2: NRQCD LDMEs for the charmonium production in Ref. 24], all in units of $\mathrm{GeV}^{3}$.

With the information of LDMEs, the direct production cross sections of $J / \psi, \psi(2 S)$ and three $\chi_{c J}$ states can be calculated as shown in Eq.(1). Furthermore, taking into account the direct production of $J / \psi$ and 
the feed-down from hadronic decays of $\psi(2 S)$ and radiative decays of three $\chi_{c J}$ states, the total $J / \psi$ cross section is estimated as follows,

$$
\sigma_{J / \psi}=\sigma_{J / \psi}^{\text {direct }}+\operatorname{Br}(\psi(2 S) \rightarrow J / \psi X) \sigma_{\psi(2 S)}+\sum_{J=0}^{2} \operatorname{Br}\left(\chi_{c J} \rightarrow J / \psi \gamma\right) \sigma_{\chi_{c J}}
$$

For the branching ratios we take the PDG 2020 values 32

$$
\begin{aligned}
\operatorname{Br}(\psi(2 S) \rightarrow J / \psi X) & =61.4 \%, & & \operatorname{Br}\left(\chi_{c 0} \rightarrow J / \psi \gamma\right)=1.4 \% \\
\operatorname{Br}\left(\chi_{c 1} \rightarrow J / \psi \gamma\right) & =34.3 \%, & & \operatorname{Br}\left(\chi_{c 2} \rightarrow J / \psi \gamma\right)=19.0 \%
\end{aligned}
$$

Using this NRQCD framework, we calculate below the $J / \psi$ and $\psi(2 S)$ production cross sections and compare them to the available fixed-target data. We explore if a set of LDMEs can be identified to achieve a good description of both proton-induced and pion-induced data of charmonia production.

\section{Results of NRQCD calculations}

In this section, we compare our NRQCD calculations with the fixed-target data of $J / \psi$ and $\psi(2 S)$ production by proton and pion beams. The cross sections for proton-induced $J / \psi$ and $\psi(2 S)$ and their ratios $R_{\psi}=\sigma(\psi(2 S)) / \sigma(J / \psi)$ are taken from Ref. [27]. The pion-induced data for $J / \psi$ and $\psi(2 S)$ are taken from Refs. [1, 33. In addition, three measurements of $R_{\psi}$ from HERA [34] and NA38 [35], and the pion-induced data of $\sigma(J / \psi)$ and $\sigma(\psi(2 S))$ from NA38 [36] and WA92 [37] are added.

In spite of a caveat of double counting by including the data of $R_{\psi}$ in the analysis [27, the cancellation of systematic uncertainties in this quantity brings additional constraining power in the fit. The NRQCD calculations presented in this section are performed using the nucleon CT14nlo PDFs [38] and the pion GRV NLO PDFs 22] under the LHAPDF framework [39, 40]. The cross sections are evaluated with a charm quark mass $m_{c}=1.5 \mathrm{GeV} / c^{2}$ and renormalization and factorization scale $\mu_{R}=\mu_{F}=2 m_{c}$ [24].

\subsection{Reference fit}

We compare these data with the NRQCD calculations using the LDMEs determined in Ref. [24], named as "Fit-R" in the following. The difference between the proton PDFs selected for the study of Ref. [24] (CTEQ3L) and here (CT14nlo) has a negligible effect on the final results. The results are plotted as black dashed lines in Fig. 1. The total reduced $\chi^{2} /$ ndf, together with the deviations between data and calculations per data point $\left(\chi^{2} / \mathrm{ndp}\right)$ for each data set, are displayed in Fig. 1 and listed in Table 3 . The reduced $\chi^{2} / \mathrm{ndf}$ of the entire data sets is 16.8. Clearly, the pion-induced data below $\sqrt{s}=20 \mathrm{GeV}$ are significantly underestimated by the calculations as already observed in Ref. [24]. 

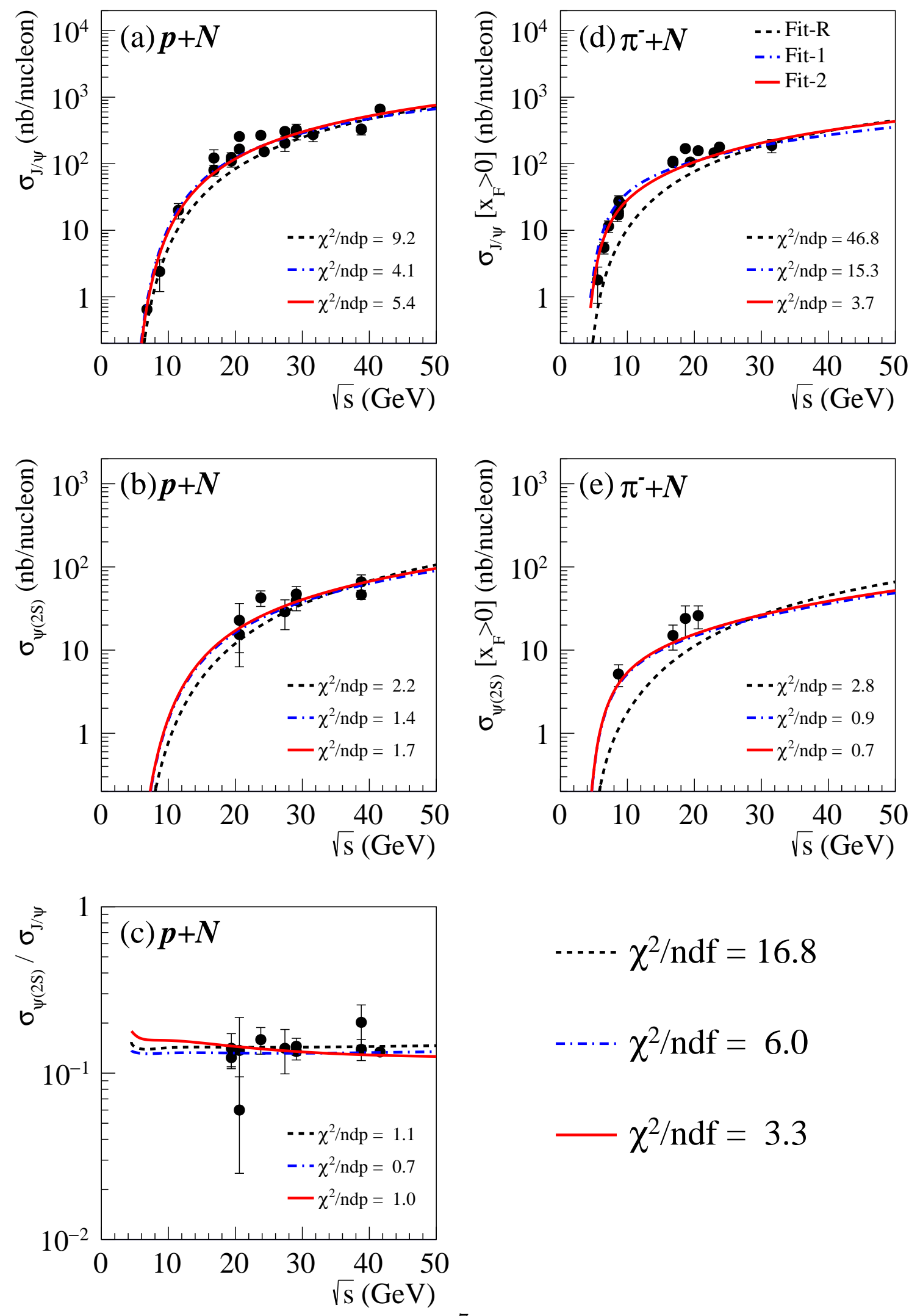

$\chi^{2} /$ ndf $=16.8$

$\chi^{2} / \mathrm{ndf}=6.0$

$\chi^{2} / \mathrm{ndf}=3.3$

Figure 1: $J / \psi$ and $\psi(2 S)$ production cross sections and the $\psi(2 S) /(J / \psi)$ ratios in the $p+N$ reactions, and $J / \psi$ and $\psi(2 S)$ production cross sections in the $\pi^{-}+N$ reactions, labeled as (a)-(e) in the plot. The dashed (black), dot-dashed (blue) and solid (red) curves represent the NRQCD results using the LDMEs obtained in "Fit-R", "Fit-1" and "Fit-2", respectively. The reduced $\chi^{2} /$ ndf for all data are displayed in the bottom-right. The values of $\chi^{2}$ divided by the number of data point (ndp) for each data set are also shown. 


\begin{tabular}{|c|c|c|c|}
\hline & Fit-R & Fit-1 & Fit-2 \\
\hline$\chi_{\text {total }}^{2} /$ ndf & 16.8 & 6.0 & 3.3 \\
\hline$\chi^{2} /\left.\mathrm{ndp}\right|_{\sigma(J / \psi)} ^{p}$ & 9.2 & 4.1 & 5.4 \\
$\chi^{2} /\left.\mathrm{ndp}\right|_{\sigma(\psi(2 S))} ^{p}$ & 2.2 & 1.4 & 1.7 \\
$\chi^{2} /\left.\mathrm{ndp}\right|_{R(\psi(2 S))} ^{p}$ & 1.1 & 0.7 & 1.0 \\
\hline$\chi^{2} /\left.\mathrm{ndp}\right|_{\sigma(J / \psi)} ^{\pi^{-}}$ & 46.8 & 15.3 & 3.7 \\
$\chi^{2} /\left.\mathrm{ndp}\right|_{\sigma(\psi(2 S))} ^{\pi^{-}}$ & 2.8 & 0.9 & 0.7 \\
\hline$\left\langle\mathcal{O}_{8}^{J / \psi}\left[{ }^{3} S_{1}\right]\right\rangle$ & $6.6 \times 10^{-3}$ & $(1.47 \pm 0.07) \times 10^{-1}$ & $(9.5 \pm 0.4) \times 10^{-2}$ \\
$\Delta_{8}^{J / \psi}$ & $3 \times 10^{-2}$ & $(0 \pm 8) \times 10^{-4}$ & $(1.8 \pm 0.2) \times 10^{-2}$ \\
$\left\langle\mathcal{O}_{8}^{\psi(2 S)}\left[{ }^{3} S_{1}\right]\right\rangle$ & $4.6 \times 10^{-3}$ & $(2.5 \pm 0.2) \times 10^{-2}$ & $(2.6 \pm 0.2) \times 10^{-2}$ \\
$\Delta_{8}^{\psi(2 S)}$ & $5.2 \times 10^{-3}$ & $(0 \pm 8) \times 10^{-4}$ & $(4 \pm 6) \times 10^{-4}$ \\
\hline
\end{tabular}

Table 3: The reduced $\chi^{2} /$ ndf of the whole data sets, the $\chi^{2}$ divided by the number of data point (ndp) for each data set in "Fit-R", "Fit-1" and "Fit-2" NRQCD calculations and the corresponding input or best-fit LDMEs. All LDMEs are in units of $\mathrm{GeV}^{3}$.

\subsection{Fit of the proton-induced data}

It is known that the $q \bar{q}$ contribution plays an important role in the pion-induced $J / \psi$ production near threshold because the parton density at large $x$ is dominated by the valence antiquarks in pions [19]. In our NRQCD framework, the $\left\langle\mathcal{O}_{8}^{H}\left[{ }^{3} S_{1}\right]\right\rangle$ LDMEs are responsible for the $q \bar{q}$ contribution, as shown in Table 1 , and their values were taken from the fits to the Tevatron data [26] at high energies. The observed underestimation of low-energy pion-induced data in "Fit-R" could arise from too small a value for the input $\left\langle\mathcal{O}_{8}^{H}\left[{ }^{3} S_{1}\right]\right\rangle$.

To seek an improved description of the pion-induced data, we take a different approach, called "Fit-1", of leaving both $\left\langle\mathcal{O}_{8}^{H}\left[{ }^{3} S_{1}\right]\right\rangle$ and $\Delta_{8}^{H}$ LDMEs for $J / \psi$ and $\psi(2 S)$ as free parameters in the global fit of the proton-induced data. The CO LDME of $\chi_{c 0}$ is fixed at the value given in Ref. [24] and the best-fit LDMEs are required to be positive-definite. The resulting fit is shown as blue dot-dashed lines in Fig. 1 . The cross sections for both proton- and pion-induced data are significantly enhanced, compared to the results of "Fit-R". The agreement between the data and calculation is greatly improved. As shown in Table 3 . the overall $\chi^{2} / \mathrm{ndf}$ is reduced from 16.8 to 6.0 , compared to "Fit-R". While the pion-induced data are not used in the global fit for the LDMEs determination, these data are included in the evaluation of $\chi^{2} /$ ndf for comparison purposes.

More specifically, the agreement between the pion-induced $J / \psi$ data alone and the calculations with bestfit LDMEs of "Fit-1" is improved by a factor of 3, compared to "Fit-R". The values of newly determined $\left\langle\mathcal{O}_{8}^{H}\left[{ }^{3} S_{1}\right]\right\rangle$ LDMEs are $1.5 \times 10^{-1}$ and $2.5 \times 10^{-2}$ for $J / \psi$ and $\psi(2 S)$, respectively. Both are significantly larger than the "Fit-R" values of $6.6 \times 10^{-3}$ and $4.6 \times 10^{-3}$ determined from collider data. The increase of the values of $\left\langle\mathcal{O}_{8}^{H}\left[{ }^{3} S_{1}\right]\right\rangle$ clearly accounts for better agreement between the NRQCD calculation and the 
pion-induced data, even though the pion data were not included in the fit.

The values of the $\mathrm{CO} \Delta_{8}^{H}$ LDMEs for $J / \psi$ and $\psi(2 S)$ resulting from "Fit-1" are compatible with zero, as shown in Table 3 . Despite an improved description of data in this approach, "Fit-1" finds vanishing values of $\Delta_{8}^{H}$ for both $J / \psi$ and $\psi(2 S)$. It appears that these LDMEs cannot be determined from the proton-induced $J / \psi$ and $\psi(2 S)$ production data alone. This suggests the need to include also the pion data in the global fit, as discussed next.

\subsection{Fit of both the pion- and proton-induced data}

Because of the different nature of valence quarks in the protons and pions, the energy dependence of the relative contributions of $q \bar{q}$ and $G G$ processes is different for the $J / \psi$ and $\psi(2 S)$ production, especially at low energies. Under the assumption that higher-twist effects are negligible, a combined fit of these two data sets should further constrain the CO LDMEs. The results of this new fit, referred to as "Fit-2", are shown in Table 3 and displayed as the solid red lines in Fig. 1 .

Comparing the results of "Fit-2" and "Fit-1", it is found that the description of the pion-induced data is improved, while maintaining a good agreement between the proton data and the calculation. The CO matrix elements are also better constrained. The total reduced $\chi^{2} /$ ndf is further decreased to about 3.3. The agreement between the pion-induced $J / \psi$ data and the NRQCD calculation is improved by a factor of 4 , from a $\chi^{2} /$ ndp of 15.3 in "Fit-1" to 3.7 in "Fit-2". The values of the newly determined CO $\left\langle\mathcal{O}_{8}^{H}\left[{ }^{3} S_{1}\right]\right\rangle$ LDMEs are $9.5 \times 10^{-2}$ and $2.6 \times 10^{-2}$ for $J / \psi$ and $\psi(2 S)$, respectively, either smaller than or consistent with those obtained in "Fit-1".

With the inclusion of the pion-induced data, non-zero values of the $\mathrm{CO} \Delta_{8}^{H}$ LDMEs can now be obtained. As shown in Table 3 , the values of $\mathrm{CO} \Delta_{8}^{H}$ LDMEs are found to be $1.8 \times 10^{-2}$ and $4.0 \times 10^{-4}$ for $J / \psi$ and $\psi(2 S)$, respectively. The best-fit $\left\langle\mathcal{O}_{8}^{H}\left[{ }^{3} S_{1}\right]\right\rangle$ LDMEs responsible for the contribution of the $q \bar{q}$ process are larger by about a factor of 10 , while the $\Delta_{8}^{H}$ related to the contribution of the $G G$ process are reduced by a factor of 2-10, in comparison with the LDMEs determined from collider data [26, 29]. The new CO LDMEs indicate that the $q \bar{q}$ contribution determined by the fixed-target data is significantly larger than the corresponding contribution at collider energies.

The systematic uncertainties of these results are studied by setting $m_{c}$ to $1.2 \mathrm{GeV} / c^{2}$ or $1.5 \mathrm{GeV} / c^{2}$ and the normalization scale $\mu=\mu_{R}=\mu_{R}$ to $m_{c}, 2 m_{c}$, and $3 m_{c}$. Figure 2 shows the comparison of the pioninduced $J / \psi$ data and NRQCD calculation with the corresponding settings of $m_{c}$ and $\mu$. The total cross sections and the $q \bar{q}, G G$, and $q G$ contributions are denoted as black, blue, red and green lines, respectively.

The parameter of the charm quark mass $m_{c}$ plays a significant role in the systematic effect. With $m_{c}$ set to $1.2 \mathrm{GeV} / c^{2}$, the CO LDMEs as free parameters are not well constrained, and the quality of fit significantly deteriorates as seen from the increased $\chi^{2} /$ ndp. Judging from the contributions of various subprocesses, the $G G$ process is enhanced too much to provide a good description of data in the calculations with this reduction 


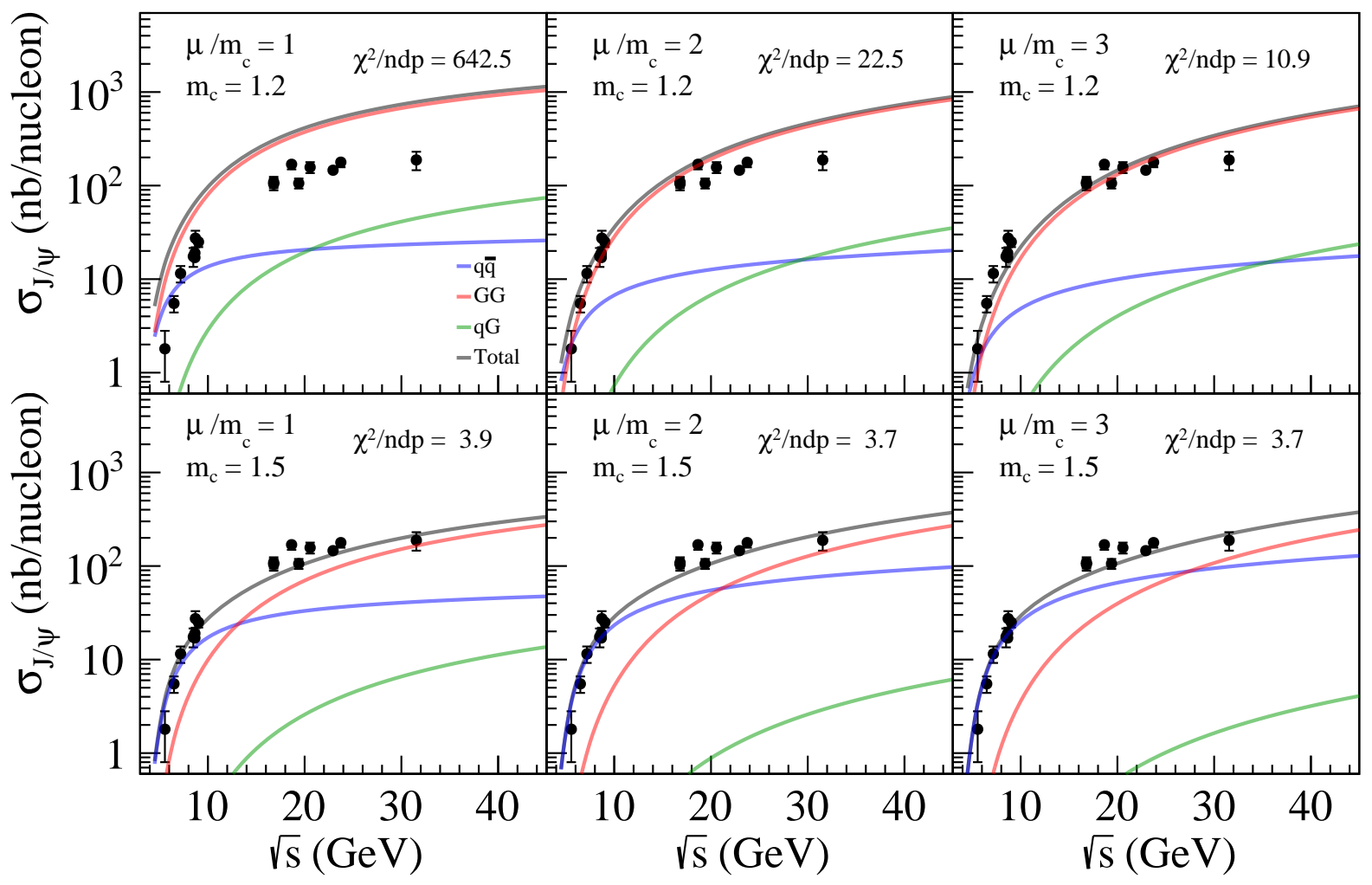

Figure 2: The NRQCD results from an analysis of both proton-induced and pion-induced data sets under variation of charm quark mass $m_{c}$, renormalization scale $\mu_{R}$ and factorization scale $\mu_{F}$, compared with the pion-induced data of $J / \psi$ production as a function of $\sqrt{s}$. The total cross sections and $q \bar{q}, G G$, and $q G$ contributions are denoted as black, blue, red and green lines, respectively. The values of $m_{c}, \mu=\mu_{R}=\mu_{F}$ in the NRQCD calculation as well as the best-fit $\chi^{2} /$ ndp are displayed in each plot. 
of $m_{c}$. When $m_{c}$ is set as $1.5 \mathrm{GeV} / c^{2}$, the quality of the fit is equally good with $\mu$ varying among $m_{c}, 2 m_{c}$, and $3 m_{c}$. Even though one of CO LDMEs cannot be obtained with good accuracy when $\mu$ is set at $m_{c}$, the best-fit LDMEs for three different scales are consistent with having large values of $\left\langle\mathcal{O}_{8}^{H}\left[{ }^{3} S_{1}\right]\right\rangle$ LDMEs, reflecting a non-negligible $q \bar{q}$ contribution.

\section{Discussion}

\subsection{Fractions of individual contributions in hadroproduction}

A new set of LDMEs for $J / \psi$ and $\psi(2 S)$ production at fixed-target energies has been obtained in an analysis of data with proton and pion beams. Our analysis differs from that of Ref. 24] in two aspects. First, the LDMEs $\left\langle\mathcal{O}_{8}^{H}\left[{ }^{3} S_{1}\right]\right\rangle$ are now allowed to vary in the global fit. This leads to a much improved description of fixed-target data with proton beam. Second, the pion data are included in the global fit. This allows for the determination of the LDMEs $\Delta_{8}^{H}$.

To better understand the reasons for the significantly improved description of the $J / \psi$ and $\psi(2 S)$ production data, it is instructive to compare the NRQCD calculations using the "Fit-R" LDMEs of Ref. 24] and the "Fit-2" LDMEs of the present analysis. In particular, we examine the decomposition of the $J / \psi$ production cross section into individual contributions in three fashions: (i) $q \bar{q}, G G$, and $q G$ subprocesses; (ii) color singlet versus color octet $c \bar{c}$ states; (iii) direct production of $J / \psi$ versus feed-down from $\psi(2 S)$ and $\chi_{c}$.

The decomposition of $J / \psi$ production cross sections for proton and pion beams into the $q \bar{q}, G G$ and $q G$ processes is shown in Fig. 3 . We note that the $q G$ contributions remain unchanged in the new analysis since the $q G$ process only contributes to the formation of the $\chi_{c 1}$ states and the LDMEs for $\chi_{c J}$ are identical for "Fit-R" and "Fit-2". Figure 3 also shows that the $G G$ contribution is dominant in the $J / \psi$ production with proton beam at all energies, except near the threshold. In contrast, the $q \bar{q}$ contribution for pion-induced data is enhanced due to the increased antiquark content in pion's valence region. Therefore, the inclusion of the pion data in the global fit provides additional constraints on those LDMEs which are sensitive to the $q \bar{q}$ process. The low-energy fixed-target pion data are particularly important for the determination of the $\left\langle\mathcal{O}_{8}^{H}\left[{ }^{3} S_{1}\right]\right\rangle$ LDMEs.

In comparison with "Fit-R", the $\left\langle\mathcal{O}_{8}^{H}\left[{ }^{3} S_{1}\right]\right\rangle$ LDME is increased, whereas $\Delta_{8}^{H}$ is decreased. These changes lead to an enhancement of the $\mathrm{CO} q \bar{q}$ contribution and a reduction of the $\mathrm{CO} G G$ contribution. The increase of the fraction of $q \bar{q}$ contribution, especially at low-energies, accounts for the improvement in describing the pion data. The opposite trend in the variations of the two CO LDMEs $\left\langle\mathcal{O}_{8}^{H}\left[{ }^{3} S_{1}\right]\right\rangle$ and $\Delta_{8}^{H}$ leads to significant changes in the energy dependence of $J / \psi$ production cross sections, as shown in Fig. 3 .

Figure 4 shows the decomposition of the $J / \psi$ cross sections into contributions from the color octet and color singlet states. As the CS contribution (blue lines) in our study is fixed, the enlarged $\left\langle\mathcal{O}_{8}^{H}\left[{ }^{3} S_{1}\right]\right\rangle$ LDMEs 

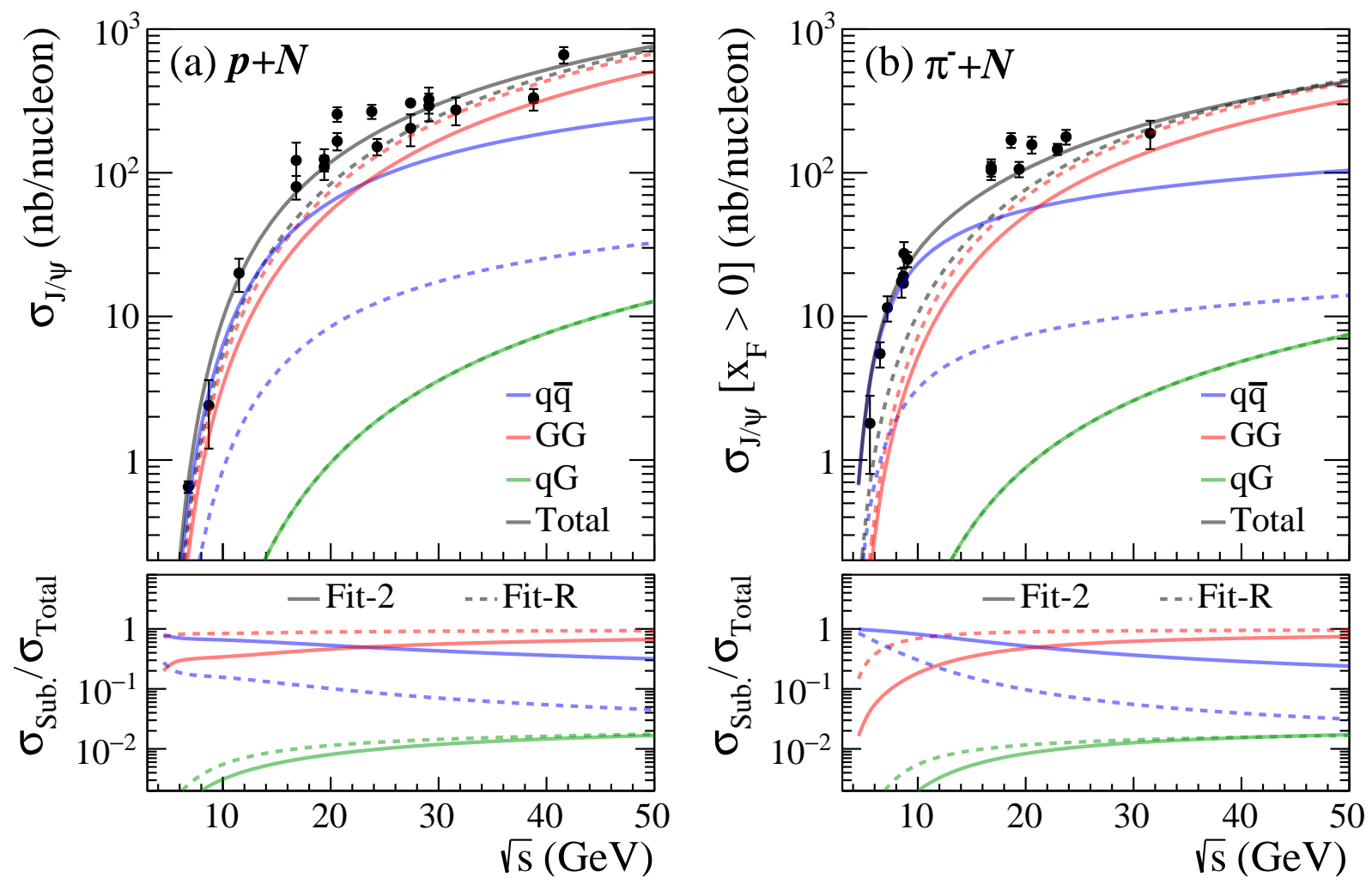

Figure 3: Total cross sections (black) and contributions from $q \bar{q}$ (blue), $G G$ (red) and $q G$ (green) processes for $J / \psi$ production as a function of $\sqrt{s}$ in (a) $p N$ and (b) $\pi^{-} N$ interactions. The dashed and solid curves represent the "Fit-R" and "Fit-2" results. The fractions of each sub-process cross section are displayed at the bottom of each plot. 

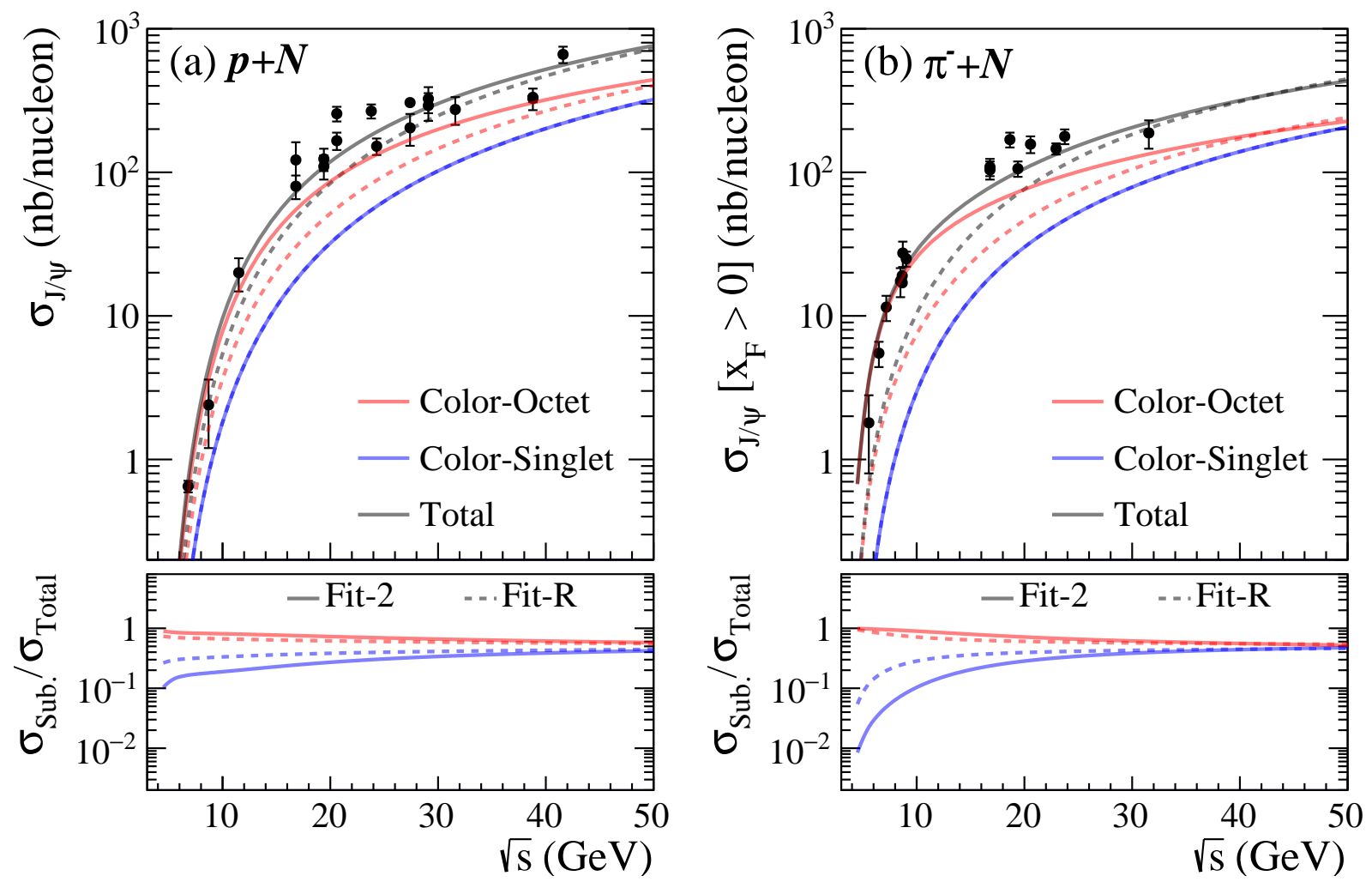

Figure 4: Same as Fig. 3 for the decomposition of contributions from CS (blue) and CO (red) processes. 
significantly enhance the $\mathrm{CO}$ contribution (red lines) at low energies, while in "Fit-2" the reduced $\Delta_{8}^{H}$ results in a reduction of the $\mathrm{CO}$ contribution at high energies. Through the interplay of these two CO LDMEs and the parton luminosities, the CO contribution remains similar at high energies but is enhanced near threshold for the proton-induced production. In the case of pion-induced production, the CO contribution is slightly suppressed at high energies.
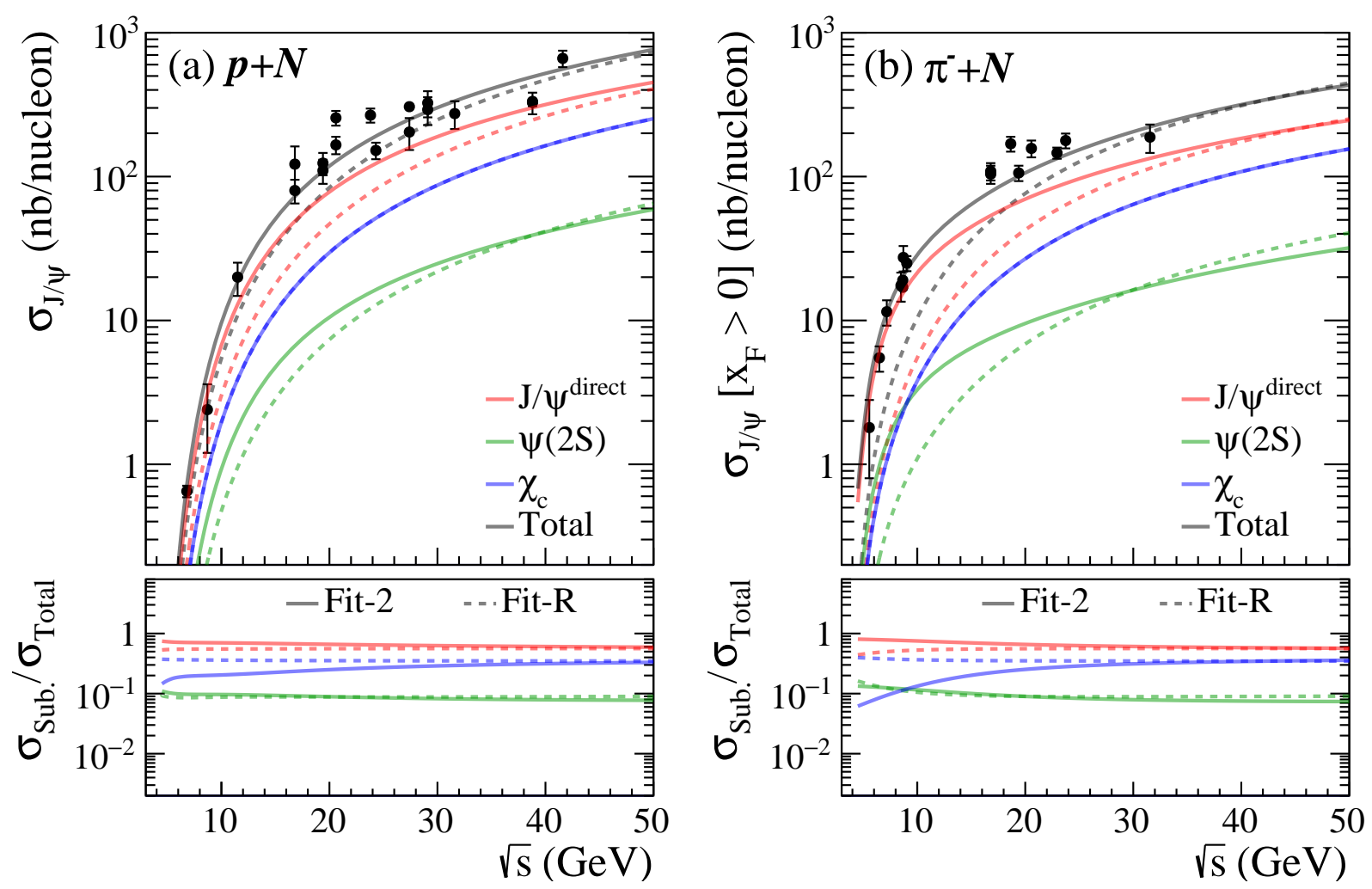

Figure 5: Same as Fig. 3 for the decomposition of contributions of $J / \psi$ production from direct production of $J / \psi$ (red) and feed-down from $\psi(2 S)$ (green) and all $\chi_{c}$ states (blue).

Figure 5 shows the decomposition of the $J / \psi$ cross sections into the contributions from direct production (red lines) and the feed-down from heavier charmonium states of $\psi(2 S)$ (blue lines) and $\chi_{c}$ (green lines). We note that the LDMEs for the three $\chi_{c}$ states are kept unchanged for "Fit-R" and "Fit-2". The most notable change between the calculations with "Fit-2" and the "Fit-R" is the enhancement of the direct $J / \psi$ production at low energies, as a consequence of an enlarged $\left\langle\mathcal{O}_{8}^{H}\left[{ }^{3} S_{1}\right]\right\rangle$. Taking into account the decay branching ratios, the contributions to the $J / \psi$ production in descending order of importance are direct production, $\chi_{c}$, and $\psi(2 S)$.

To recapitulate the main findings at this point, we note that the inclusion of the low-energy pion-induced total cross section data of $J / \psi$ and $\psi(2 S)$ production to the analysis of NRQCD provides an important constraint of the $\left\langle\mathcal{O}_{8}^{H}\left[{ }^{3} S_{1}\right]\right\rangle$ LDMEs via the $q \bar{q}$ contributions. A good description of both proton- and pion- 
induced data by NRQCD can be achieved. The $q \bar{q}$ and CO contributions from the NRQCD calculations with the new LDMEs are greatly enhanced at low energies with proton and pion beams, compared with results found in the earlier studies [24, 27].

\subsection{Sensitivity to the pion PDFs}

The new set of LDMEs is now used to study the sensitivity of the $J / \psi$ data to the various pion PDFs. We have considered four pion PDFs, namely, SMRS [21] and GRV [22], representative of the most widely used pion PDFs thus far, as well as JAM [6] and xFitter [7, obtained from very recent global analyses. For SMRS, we select the default one in which the sea quarks carry $15 \%$ of the pion momentum at $Q^{2}=4$ $\mathrm{GeV}^{2}$. As illustrated in Ref. [19], SMRS, JAM, and xFitter have similar valence-quark distributions, while the magnitude of the GRV distribution is smaller by about 20\%-30\%. For the gluon distributions, SMRS and GRV have similar shapes and magnitudes, while xFitter and JAM have significantly smaller magnitudes by a factor of $2-4$.

The NRQCD calculation with each of the four pion PDFs is compared with the data in Fig. 6. Overall, the total cross sections (black lines) for the four pion PDFs exhibit similar $\sqrt{s}$ dependencies. However, the individual terms differ strongly. The $q \bar{q}$ contribution dominates near thresholds and the $G G$ contribution increases rapidly at higher energies, while the $q G$ component is relatively negligible over the whole energy range. The relative fractions of $q \bar{q}$ and $G G$ contributions as a function of $\sqrt{s}$ vary for each pion PDFs, reflecting the differences among their parton distributions. For SMRS and GRV the $G G$ contribution starts to dominate the cross section around $\sqrt{s}=20 \mathrm{GeV}$. For xFitter and JAM the corresponding values are larger at $\sim \sqrt{s}=35 \mathrm{GeV}$ because of their relatively reduced gluon strength in the valence region.

\begin{tabular}{|c|c|c|c|c|}
\hline & SMRS & GRV & JAM & xFitter \\
\hline$\chi_{\text {total }}^{2} / \mathrm{ndf}$ & 3.1 & 3.4 & 4.8 & 4.3 \\
\hline$\chi^{2} /\left.\mathrm{ndp}\right|_{\sigma(J / \psi)} ^{\pi^{-}}$ & 2.7 & 3.7 & 8.4 & 6.4 \\
$\chi^{2} /\left.\mathrm{ndp}\right|_{\sigma(\psi(2 S))} ^{\pi^{-}}$ & 0.4 & 0.7 & 0.4 & 0.2 \\
\hline$\left\langle\mathcal{O}_{8}^{J / \psi}\left[{ }^{3} S_{1}\right]\right\rangle$ & $(6.9 \pm 0.3) \times 10^{-2}$ & $(9.5 \pm 0.4) \times 10^{-2}$ & $(8.3 \pm 0.3) \times 10^{-2}$ & $(7.4 \pm 0.9) \times 10^{-2}$ \\
$\Delta_{8}^{J / \psi}$ & $(2.5 \pm 0.2) \times 10^{-2}$ & $(1.8 \pm 0.2) \times 10^{-2}$ & $(2.0 \pm 0.2) \times 10^{-2}$ & $(2.2 \pm 0.2) \times 10^{-2}$ \\
$\left\langle\mathcal{O}_{8}^{\psi(2 S)}\left[{ }^{3} S_{1}\right]\right\rangle$ & $(2.1 \pm 0.4) \times 10^{-2}$ & $(2.6 \pm 0.2) \times 10^{-2}$ & $(2.6 \pm 0.1) \times 10^{-2}$ & $(2.3 \pm 0.4) \times 10^{-2}$ \\
$\Delta_{8}^{\psi(2 S)}$ & $(1.7 \pm 1.0) \times 10^{-3}$ & $(4.0 \pm 6.2) \times 10^{-4}$ & $(3.7 \pm 3.4) \times 10^{-4}$ & $(0.9 \pm 6.0) \times 10^{-3}$ \\
\hline
\end{tabular}

Table 4: The $\chi^{2}$ values for the entire data sets and the individual $J / \psi$ and $\psi(2 S)$ data set from the NRQCD calculations. The best-fit LDMEs for each pion PDF are in units of $\mathrm{GeV}^{3}$.

Table 4 lists the $\chi^{2}$ values of NRQCD calculations for various data sets and best-fit CO LDMEs for each pion PDF. We find that the $\chi^{2}$ of the pion-induced $J / \psi$ data strongly correlates with the gluon density of pions over the valence quark regions [19]. The $G G$ contributions are similar for GRV and SMRS, while those 

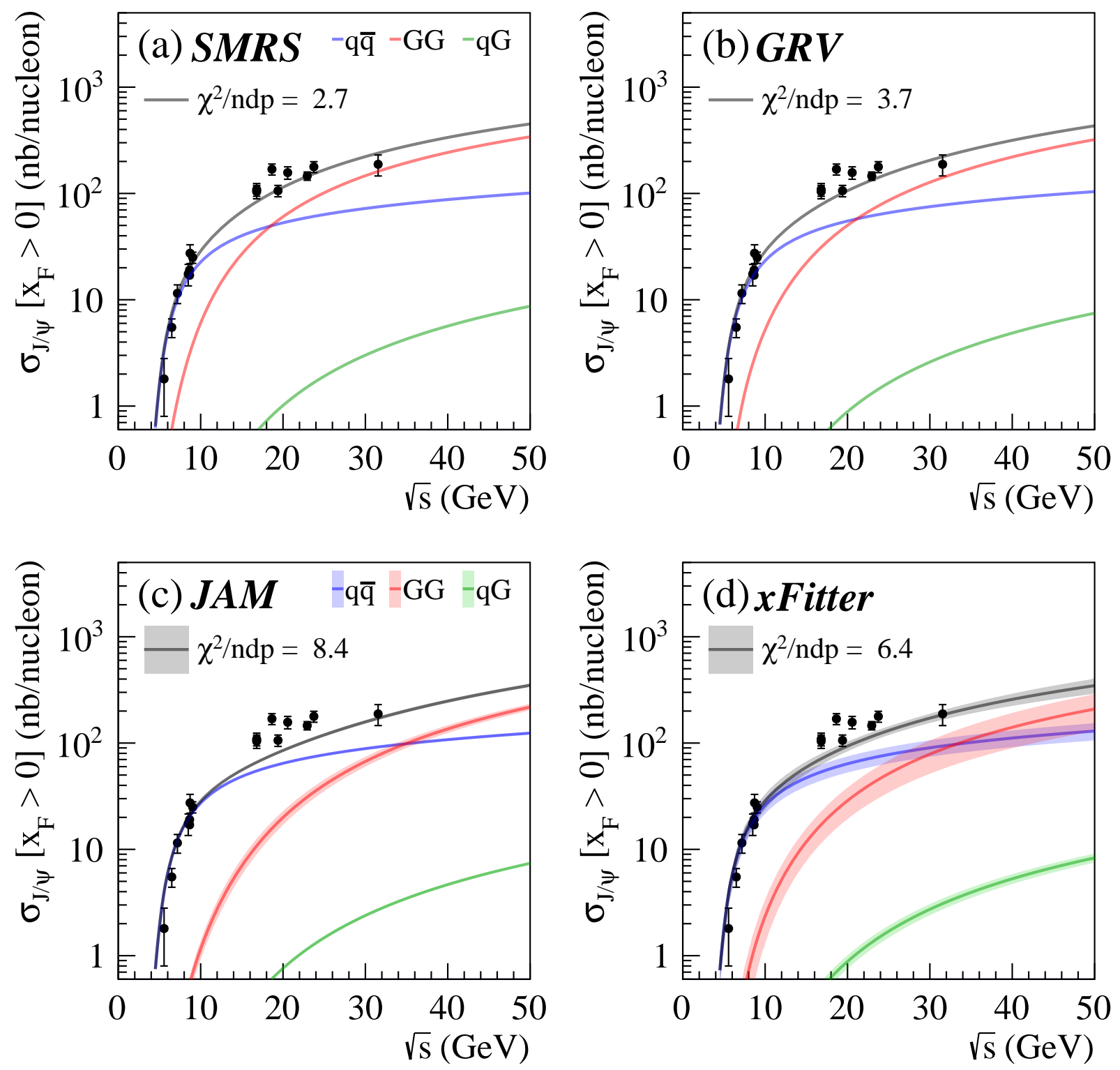

Figure 6: The NRQCD $J / \psi$ production cross sections at $x_{F}>0$ for the $\pi^{-} N$ reactions, calculated for four pion PDFs (SMRS, GRV, JAM and xFitter) using LDMEs of "Fit-2". The black, blue, red, and green curves represent the calculated total cross sections, and the $q \bar{q}, G G$, and $q G$ contributions, respectively. The shaded bands on the xFitter and JAM calculations come from the uncertainties of the corresponding PDF sets. The SMRS and GRV PDFs contain no information on uncertainties. 
for xFitter and JAM are 50-80\% smaller due to their weaker gluon strength at $x=0.1-0.6$, relative to GRV and SMRS [19]. The deficiency of xFitter and JAM in the $G G$ contribution leads to an underestimation of the NRQCD calculations against the data over $\sqrt{s}=15-25 \mathrm{GeV}$, as shown in Fig. 6 .

Table 4 shows that the dependence of the best-fit LDMEs for $J / \psi$ and $\psi(2 S)$ to the pion PDFs is rather mild. We also checked that the overall reduced $\chi^{2} /$ ndf of data for each pion PDF has a very small variation when the calculations are done with the best-fit LDMEs obtained using a different set of pion PDFs.

\section{Summary}

We have analyzed $J / \psi$ and $\psi(2 S)$ hadroproduction data in fixed-target experiments within the framework of NRQCD. The previously reported difficulty [24] of obtaining a consistent description of proton and pion data by NRQCD, is resolved by allowing the CO LDMEs for $J / \psi$ and $\psi(2 S)$ to be determined by a simultaneous fit to both proton- and pion-induced data. The pion-induced data, especially at low energies, have a sizable $q \bar{q}$ contribution and thus provide a strong constraint on the $\left\langle\mathcal{O}_{8}^{H}\left[{ }^{3} S_{1}\right]\right\rangle$ LDMEs. This sensitivity is much reduced if the analysis is restricted to the proton-induced data alone. Consequently, the best-fit $\left\langle\mathcal{O}_{8}^{H}\left[{ }^{3} S_{1}\right]\right\rangle$ values for both $J / \psi$ and $\psi(2 S)$ are found to be about $\sim 10$ times larger than that same values obtained using the collider data. In contrast, the best-fit values for $\Delta_{8}^{H}$, partly responsible for the $G G$ contribution, are compatible for $J / \psi$ but more than 10 times smaller for $\psi(2 S)$, compared to the results of Ref. [24].

The resulting LDMEs combined with four different pion PDFs were used to compare the NRQCD calculation with the pion-induced $J / \psi$ production cross-sections. The pronounced differences between the predicted individual quark-antiquark annihilation and gluon-gluon fusion terms result from the different shapes and magnitudes of the corresponding PDF parametrizations. When compared to the total cross section, the SMRS or GRV PDFs still provide a slightly better description of the data than JAM or xFitter, suggesting that the data favor those PDFs with larger gluon contents at medium and large $x$. All these results are in line with our earlier findings [19] obtained with the CEM.

We note that there are the recent state-of-the-art NLO NRQCD fits [41, 42, 43, 44] to collider data. The $\left\langle\mathcal{O}_{8}^{J / \psi}\left[{ }^{3} S_{1}\right]\right\rangle$ LDMEs found in Refs. [41, 43] are substantially smaller than our results. In the fixed-target domain considered here the mean $p_{T}$ values are lower than the charmonium mass. In this low- $p_{T}$ region the factorization assumption of NRQCD may no longer be valid. Higher-order corrections or other additional contributions could therefore spoil the universality of the LDMEs. It will be interesting to perform similar studies, including the pion-induced data of charmonium production with the full NLO NRQCD formalism and make a comparison with the current results in the future.

In the near future, new measurements of Drell-Yan as well as $J / \psi$ data in $\pi^{-} N$ reactions will be available from the CERN COMPASS [4] and AMBER [46] experiments. While future theoretical advances are needed 
to further reduce the model dependence in describing the $J / \psi$ production, we emphasize the importance of including the pion-induced $J / \psi$ data in future pion PDF global analysis.

\section{Acknowledgments}

We thank Nobuo Sato and Ivan Novikov for providing us with LHAPDF6-compatible grid files of JAM and xFitter PDFs. This work was supported in part by the U.S. National Science Foundation (Grant PHY18-22502) and the Ministry of Science and Technology of Taiwan.

\section{References}

[1] G. A. Schuler, Quarkonium production and decays, arXiv:hep-ph/9403387

[2] G. A. Schuler, Production of heavy quarks and heavy quarkonia, Z. Phys. C 71 (1996) 317.

[3] N. Brambilla et al., Heavy Quarkonium: Progress, Puzzles, and Opportunities, Eur. Phys. J. C 71 (2011) 1534.

[4] A. Andronic et al., Heavy-flavour and quarkonium production in the LHC era: from proton-proton to heavy-ion collisions, Eur. Phys. J. C $\mathbf{7 6}$ (2016) 107.

[5] J. P. Lansberg, New Observables in Inclusive Production of Quarkonia Phys. Rept. 889 (2020) 1.

[6] P. C. Barry, N. Sato, W. Melnitchouk, and C. R. Ji, First Monte Carlo Global QCD Analysis of Pion Parton Distributions, Phys. Rev. Lett. 121 (2018) 152001.

[7] I. Novikov et al., Parton Distribution Functions of the Charged Pion Within The xFitter Framework, Phys. Rev. D 102 (2020) 014040 .

[8] C. Bourrely, F. Buccella and J. C. Peng, A new extraction of pion parton distributions in the statistical model, Phys. Lett. $B 813$ (2021) 136021.

[9] K. D. Bednar, I. C. Cloët, and P. C. Tandy, Distinguishing Quarks and Gluons in Pion and Kaon PDFs, Phys. Rev. Lett. 124 (2020) 042002.

[10] Z. F. Cui et al., Kaon and pion parton distributions, Eur. Phys. J. C 80 (2020) 1064.

[11] G. F. de Teramond et al. [HLFHS Collaboration], Universality of Generalized Parton Distributions in Light-Front Holographic QCD, Phys. Rev. Lett. 120 (2018) 182001 (2018).

[12] J. Lan, C. Mondal, S. Jia, X. Zhao, and J. P. Vary, Parton Distribution Functions from a Light Front Hamiltonian and QCD Evolution for Light Mesons, Phys. Rev. Lett. 122 (2019) 172001.

[13] J. H. Zhang, J. W. Chen, L. Jin, H. W. Lin, A. Schäfer, and Y. Zhao, First direct lattice-QCD calculation of the $x$ dependence of the pion parton disution function, Phys. Rev. D 100 (2019) 034505.

[14] R. S. Sufian, J. Karpie, C. Egerer, K. Orginos, J. W. Qiu, and D. G. Richards, Pion Valence Quark Distribution from Matrix Element Calculated in Lattice QCD, Phys. Rev. D 99 (2019) 074507.

[15] T. Izubuchi, L. Jin, C. Kallidonis, N. Karthik, S. Mukherjee, P. Petreczky, C. Shugert, and S. Syritsyn, Valence parton distribution function of pion from fine lattice, Phys. Rev. D 100 (2019) 034516.

[16] B. Joó, J. Karpie, K. Orginos, A. V. Radyushkin, D. G. Richards, R. S. Sufian, and S. Zafeiropoulos, Pion valence structure from Ioffe-time parton pseudodistribution functions, Phys. Rev. D 100 (2019) 114512.

[17] R. S. Sufian et al., Pion Valence Quark Distribution from Current-Current Correlation in Lattice QCD, Phys. Rev. D 102 (2020) 054508.

[18] X. Gao et al., Valence parton distribution of the pion from lattice QCD: Approaching the continuum limit, Phys. Rev. D 102 (2020) 094513. 
[19] W. C. Chang, J. C. Peng, S. Platchkov and T. Sawada, Constraining gluon density of pions at large $x$ by pion-induced $J / \psi$ production, Phys. Rev. D 102 (2020) 054024.

[20] M. B. Einhorn and S. D. Ellis, Hadronic Production of the New Resonances: Probing Gluon Distributions, Phys. Rev. D 12 (1975) 2007; H. Fritzsch, Producing Heavy Quark Flavors in Hadronic Collisions: A Test of Quantum Chromodynamics, Phys. Lett. 67B (1977) 217; F. Halzen, CVC for Gluons and Hadroproduction of Quark Flavors, Phys. Lett. 69B (1977) 105.

[21] P. J. Sutton, A. D. Martin, R. G. Roberts, and W. J. Stirling, Parton distributions for the pion extracted from Drell-Yan and prompt photon experiments, Phys. Rev. D 45 (1992) 2349.

[22] M. Gluck, E. Reya, and A. Vogt, Pionic parton distributions, Z. Phys. C 53 (1992) 651.

[23] G. T. Bodwin, E. Braaten, and G. P. Lepage, Rigorous QCD analysis of inclusive annihilation and production of heavy quarkonium, Phys. Rev. D 51 (1995) 1125; 55 (1997) 5853(E).

[24] M. Beneke and I. Z. Rothstein, Hadroproduction of quarkonia in fixed target experiments, Phys. Rev. D 54 (1996) 2005; 54 (1996) 7082(E).

[25] E. J. Eichten and C. Quigg, Quarkonium wave functions at the origin, Phys. Rev. D 52 (1995) 1726.

[26] P. L. Cho and A. K. Leibovich, Color octet quarkonia production. II, Phys. Rev. D 53 (1996) 6203.

[27] F. Maltoni et al., Analysis of charmonium production at fixed-target experiments in the NRQCD approach, Phys. Lett. B 638 (2006) 202.

[28] A. Petrelli, M. Cacciari, M. Greco, F. Maltoni and M. L. Mangano, NLO production and decay of quarkonium, Nucl. Phys. B 514 (1998) 245.

[29] M. Beneke and M. Krämer, Direct $J / \psi$ and $\psi^{\prime}$ polarization and cross-sections at the Tevatron, Phys. Rev. D 55 (1997) 5269.

[30] P. Nason et al., Bottom production, 1999 Workshop on Standard model physics (and more) at the LHC, 231-304, arXiV:hep-ph/0003142

[31] R. Vogt, The $x(F)$ dependence of $\psi$ and Drell-Yan production, Phys. Rev. C 61 (2000) 035203.

[32] P.A. Zyla et al. (Particle Data Group), Prog. Theor. Exp. Phys. 2020 (2020) 083C01.

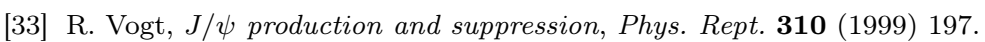

[34] I. Abt et al. [HERA-B Collaboration], A Measurement of the $\psi^{\prime}$ to J/ $\psi$ production ratio in 920-GeV proton-nleus interactions, Eur. Phys. J. C 49 (2007) 545.

[35] C. Baglin et al. [NA38 Collaboration], psi-Prime and $J / \psi$ production in $p W, p U$ and $S U$ interactions at 200GeV/nucleon, Phys. Lett. B 345 (1995) 617.

[36] M. J. Corden et al., Experimental Results on $J / \psi$ Production by $\pi^{ \pm}, K^{ \pm}, p$ and $\bar{p}$ Beams at 39.5-GeV, Phys. Lett. 96B (1980) 411.

[37] Y. Alexandrov et al. [BEATRICE Collaboration], Inclusive $J / \psi$ and $\psi^{\prime}$ production in $\pi^{-}-$nucleus interactions at $S^{(1 / 2)}$ approximately equals 26-GeV, Nucl. Phys. B $\mathbf{5 5 7}$ (1999) 3.

[38] S. Dulat et al., New parton distribution functions from a global analysis of quantum chromodynamics, Phys. Rev. D 93 (2016) 033006.

[39] M. R. Whalley, D. Bourilkov and R. C. Group, The Les Houches accord PDFs (LHAPDF) and LHAGLUE, arXiv:hep$\mathrm{ph} / 0508110$.

[40] A. Buckley, J. Ferrando, S. Lloyd, K. Nordström, B. Page, M. Rüfenacht, M. Schönherr and G. Watt, LHAPDF6: parton density access in the LHC precision era, Eur. Phys. J. C 75 (2015) 132.

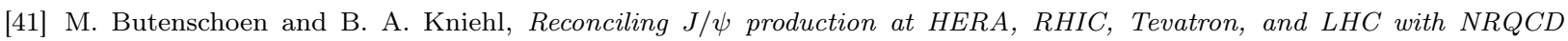
factorization at next-to-leading order, Phys. Rev. Lett. 106022003 (2011).

[42] Y. Q. Ma, K. Wang and K. T. Chao, $J / \psi\left(\psi^{\prime}\right)$ production at the Tevatron and LHC at $\mathcal{O}\left(\alpha_{s}^{4} v^{4}\right)$ in nonrelativistic QCD, 
Phys. Rev. Lett. 106 (2011) 042002.

[43] M. Butenschoen and B. A. Kniehl, World data of $J / \psi$ production consolidate NRQCD factorization at NLO, Phys. Rev. D 84 (2011) 051501.

[44] Y. Q. Ma, K. Wang and K. T. Chao, A complete NLO calculation of the $J / \psi$ and $\psi^{\prime}$ production at hadron colliders, Phys. Rev. D 84 (2011) 114001.

[45] M. Aghasyan et al. [COMPASS Collaboration], First measurement of transverse-spin-dependent azimuthal asymmetries in the Drell-Yan process, Phys. Rev. Lett. 119 (2017) 112002; C. Y. Hsieh, JPS Conf. Proc. 26 (2019) 031003.

[46] B. Adams et al., Letter of Intent: A New QCD facility at the M2 beam line of the CERN SPS (COMPASS++/AMBER), arXiv:1808.00848 